\title{
An Analytical Study with a Novel Surgical Technique By "Traction Release ILM Peeling" for Large Diameter Chronic Macular Holes
}

\author{
Rajkumar Maheshwari ${ }^{1}$, Sajeev Cherian Jacob ${ }^{2}$, Lavanya Kalikivayi ${ }^{3}$ M, Linu Raphel ${ }^{4}$ B and Venkataramana \\ Kalikivayi*5 \\ Consultant Ophthalmologist, Ahalia Foundation Eye Hospital, India
}

Submission: December 16, 2019; Published: February 07, 2020

*Corresponding author: Venkataramana Kalikivayi, Ahalia School of Optometry, Ahalia Foundation Eye Hospital, Palakkad, Kerala, India

Abstract

Title: An Analytical Study with a novel Surgical Technique by “Traction Release ILM Peeling” for Large Diameter Chronic Macular holes.

Purpose: To analyze the effect of anatomical and functional outcome of large diameter chronic macular holes by managing through a novel Surgical Technique which includes Traction Release ILM Peeling (TRILMP) without manipulating the flap using only silicone oil tamponade.

Design: Prospective Cohort study.

Subjects: A prospective study of 15 patients having stage 4 chronic large diameter macular holes were recruited by stratified random sampling technique.

Methods: Contrary to the conventional inverse ILM peeling surgery, a modified TRILMP technique was used for all chronic macular hole cases. Surgical procedure was performed in 15 patients by a single surgeon. Functional visual outcome was measured by Log MAR visual acuity charts and anatomical closure by OCT imaging to calculate the basal hole diameter, the minimum hole diameter and hole form factor (HFF).

Main outcome measures: Both anatomical and functional factors involving hole form factor, basal hole diameter and visual improvement postoperatively were analyzed.

Results: Fourteen of 15 eyes (93\%) had type 1 closure with a mean basal hole diameter of $1249 \pm 174 \mu \mathrm{m}$ and one eye (7\%) had type 2 closure with a basal hole diameter of $1683 \mu \mathrm{m}$. A statistically significant improvement in the visual outcome with $\mathrm{p}<0.05$ was found between pre and post-operative Log MAR values. A significant negative correlation $(\mathrm{p}=0.04)$ was found between hole form factor $(\mathrm{HFF})(\mathrm{Mean}=0.54)$ and post-operative Log MAR visual acuity. Similarly, a significant positive correlation ( $\mathrm{p}=0.001)$ was found between basal hole diameter and postoperative Log MAR visual acuity. Functional visual improvement of $61 \%$ was achieved 6 months postoperatively where the maximum visual acuity improved from $\log 1.3-\log 0.3$.

Conclusion: A lone tamponade of silicone oil was used along with a novel Surgical Technique adopting traction release ILM peeling without manipulating the flap remnant around macular hole edge, which gave an enhanced anatomical and functional result even in macular holes with larger basal diameter and a medium HFF.

Keywords: ILM peeling; Silicone oil; Chronic macular hole; Hole form factor; Basal diameter.

\section{Introduction}

Idiopathic macular holes are one of the commonest causes of central vision impairment in the elderly group. The prevalence rates are $0.02 \%$ in Australia, $0.09 \%$ in China, $0.17 \%$ in southern India and $0.3 \%$ in Baltimore [1-4]. "The Eye Disease Case-Control Study" reported that more than $50 \%$ of the patients were between the age of 65-74 years and $72 \%$ of idiopathic holes occurred in women [5]. In 1988, Gass proposed that the contraction of foveolar cortical vitreous resulted in tangential traction which led to a predictable progression through multiple stages [6]. Since the 1990s, the emergence of Optical Coherence Tomography (OCT) led to a significant appreciation of the tractional forces and the sequence of events from vitreomacular traction to the formation of a full-thickness macular hole. The first calculated OCT index used as a prognostic factor was the hole form factor (HFF) [7]. It is the quotient of the summation of the left and right arm lengths divided by the basal hole diameter. The basal hole diameter is the 
diameter of the macular hole measured at the level of retinal pigment epithelium. The technique proposed by Kelly and Wendel in 1991 [8] remains the basis for the standard surgical repair of macular hole even today. This includes, 3 port pars plana vitrectomy with removal of cortical vitreous and epiretinal membrane (ERM) followed by an intraocular tamponade by $\mathrm{C}_{3} \mathrm{~F}_{8}$ gas. After the surgery, the patient had to maintain a strict face-down position for no less than a week in order to attain a maximum tamponade of the macula. Currently, several modifications of the standard technique are developed. The newer techniques include internal limiting membrane (ILM) peeling [9] and use of surgical adjuvants like TGF beta 2, autologous serum, platelets, and whole blood [8-11]. The ILM peeling around the macular hole was found to increase the success rate close to $88-100 \%$ possibly by removing all the tractional elements, [12-16] thereby aiding better hole closure. In 2010, an additional "Inverted ILM flap" technique, was put forth which was proved to increase the rate of complete closure of the macular hole with improved visual outcome [17]. This technique involved leaving remnants of ILM at the macular hole margin to about 2 disc diameters (DD). This ILM remnant was then inverted upside-down to cover the macular hole and the fluid-air exchange was performed. Studies done by Kannan et al. [18] and Chabblani et al. [19] showed that inverted ILM flap technique did not lead to significantly higher closure rates as opposed to conventional ILM peeling in large macula hole $(\mathrm{MH})>800 \mu \mathrm{m}$. In the study by Shukla et al. [20] it was found that $81 \%$ of chronic macular holes achieved anatomical closure by using ILM peeling and $\mathrm{C}_{3} \mathrm{~F}_{8}$ tamponade. It was also reported that $62 \%$ attained type 1 closure, $19 \%$ attained type 2 closure and larger holes with a mean of $1205 \mu \mathrm{m}$ remained open postoperatively. In their study, larger holes were more likely to remain open after surgical repair. Several studies had used silicone oil tamponade for treatment of idiopathic macular holes predominantly to avoid postoperative head posturing and the results achieved were comparable with C3F8 tamponade [21-23].

Lai and McCuen compared silicone oil versus gas tamponade and found that $\mathrm{C}_{3} \mathrm{~F}_{8}$ gas proved to be a more effective tamponade than silicone oil with respect to achieving initial closure of macular holes [24]. Eyes receiving an oil tamponade required significantly more reoperations to achieve a similar rate of hole closure compared with eyes undergoing a gas tamponade. Final visual acuity was better for gas-operated eyes than for silicone-operated eyes. The aim of this study was to analyze the results of a novel surgical technique for chronic macular holes with pars plana vitrectomy adopting traction release ILM peeling (TRILMP), leaving a circular remnant of $2 / 3^{\text {rd }}$ DD of ILM around the MH edges with silicone oil tamponade.

\section{Materials and Methods}

This prospective cohort study recruited patients from a single surgeon who were diagnosed to have chronic (duration $>6$ months) full-thickness stage 4 macular hole. Patients with previous history of trauma and vitreoretinal surgeries were excluded.
Institutional ethics committee approval was obtained, and this study followed the tenets of the declaration of Helsinki. Surgical procedure was performed in 15 eyes of 15 patients after obtaining written informed consent. Preoperative evaluation included objective and subjective refraction, intraocular pressure (IOP) measurement, slit lamp biomicroscopy along with retinal evaluation with indirect ophthalmoscopy. OCT imaging was performed to calculate the basal hole diameter, the minimum hole diameter and hole form factor (HFF). All the patients underwent 25G pars plana vitrectomy using Zeiss resight biome wide field lens. The removal of posterior hyaloid was aided using triamcinolone acetonide. Brilliant Blue dye was used under air for $<5$ seconds for better visualization of the ILM. Alcon ILM forceps was used to pinch and elevate the ILM. Unlike earlier works, the ILM remnant was not inverted and placed into the MH which was the customary procedure. Instead ILM peeling was performed in a doughnut-shaped fashion leaving approximately a circular remnant of $2 / 3^{\text {rd }} D D$ of ILM still attached to the margins of the macular hole. Once the traction on the edges of MH was released, hole closure was more rapid and rewarding. Exchange of fluid-air was followed by filling of silicone oil (1500cs) tamponade. Complete fill of the vitreous cavity devoid of air bubbles was ensured. Patients were followed up on day 1, 1 week, 1 month, 2 months and 6 months postoperatively. At each visit best corrected visual acuity (BCVA) and IOP were recorded. OCT imaging was done on the next day and at 1 month post op review. Silicone oil removal was done between 2-3 months. Anatomical outcome and final visual acuity were assessed for each patient at the end of 6 months follow up.

Descriptive analyses were computed for all the patients. Student's t test was performed to analyze the pre-op and post-op results. A simple linear regression was carried out to estimate the influence of basal hole diameter, minimum diameter and hole form factor on the functional visual outcome postoperatively.

\section{Results}

A total of 15 eyes from 15 patients with chronic idiopathic macular holes were included in the study. There were 6 males and 9 females in the group with an average age of $65 \pm 5.64$ years and $65 \pm 3.63$ years respectively. All the patients had a full-thickness stage 4 macular hole (according to Gass classification). OCT imaging was done to measure the basal hole diameter, the minimum hole diameter and the hole form factor (HFF) was calculated. The mean basal hole diameter in the group was $1270 \pm 203$ microns with a minimum of 851 microns and maximum of 1683 microns. The minimum hole diameter ranged between 338 to $844 \mu \mathrm{m}$ with a mean of $697 \pm 141 \mathrm{um}$. The average HFF in the group was calculated and was found to be $0.54 \pm 0.09$ with a minimum of 0.41 and a maximum of 0.77 . Fourteen of 15 eyes (93\%) had type 1 closure with a mean basal hole diameter of $1249 \pm 174 \mu \mathrm{m}$ and mean minimum hole diameter of $687 \pm 141 \mu \mathrm{m})$. One eye ( $7 \%$ ) had type 2 closure with a basal diameter of $1683 \mu \mathrm{m}$ and minimum hole diameter of $826 \mu \mathrm{m}$. The mean preoperative visual acuity was $1.15 \pm 0.17$ 
Log MAR with a range of 0.8 to 1.5 Log MAR. The mean postoperative visual acuity was $0.91 \pm 0.36$ and $0.75 \pm 0.33$ Log MAR after 2 weeks and 2 months respectively. At 6 months review, the mean visual acuity was $0.7 \pm 0.39 \mathrm{Log}$ MAR units. There was a statistically significant improvement in visual acuity between pre-operative and 2 weeks $(\mathrm{p}=0.01)$ and 2 months $(\mathrm{p}<0.001)$ postoperative Log MAR values respectively, whereas there was no statistical significance with 6 months postoperative Log MAR values.

In this study, 93\% had anatomical type 1 closure and only one case (7\%) had a type 2 closure. At 6 months follow up, functional visual improvement of $61 \%$ was achieved postoperatively. A sample OCT image, before and after macular hole surgery is depicted in Figure $1 \& 2$ respectively. There was a positive correlation with no statistical significance between HFF and preoperative Log MAR acuity, whereas a non-significant negative correlation was found between HFF and 2 weeks and 2-months postoperative Log MAR acuity. However, HFF and 6-month postoperative Log MAR showed a significant negative correlation with $p=0.04$ and $R_{2}=0.38$ as shown in Figure 3. A statistically significant positive correlation was found between basal hole diameter and 2-week postoperative Log MAR acuity with $p=0.001$ and $R_{2}=0.55$ as shown in Figure 4 . Nevertheless, a positive correlation between basal hole diameter and 2-month and 6-month postoperative Log MAR acuity was present with no statistical significance with $p>0.05$. A statistically significant positive correlation was found between minimum diameter and postoperative 2 weeks and 2-month postoperative Log MAR acuity ( $\left.p=0.008 \& 0.02 ; R_{2}=0.43 \& 0.46\right)$ as represented in Figure $5 \& 6$ respectively.

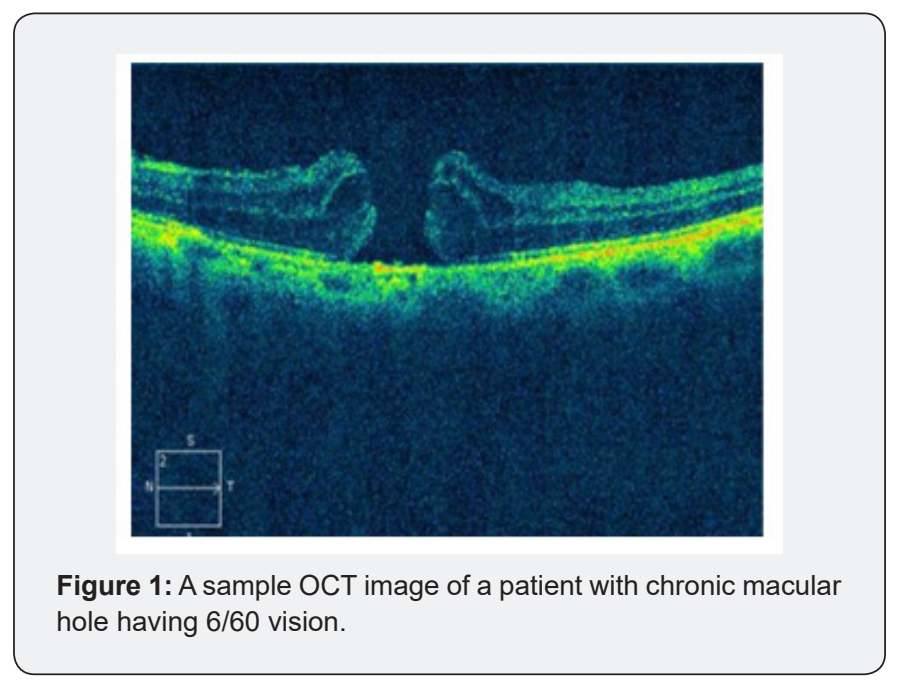

\section{Discussion}

Macular hole surgeries are constantly evolving through time as a result of various modifications attempted to the classic procedure. Earlier works $[9-11,17,25,26]$ discussed in detail about the success of surgeries with C3F8 gas tamponade which necessitate a strict postoperative face-down position. Anatomical success rate of $90 \%$ and visual improvement in $70 \%$ of the operated eyes were reported by Ivanovska Adjievska et al. [27] by combining conventional ILM peeling and silicone oil tamponade (mean pre-op BCVA was 0.8 log MAR and post-op BCVA at 3 months was 0.6). Compared with conventional ILM peeling, the inverted ILM flap technique was more effective for macular hole closure when combined with silicon oil tamponade. In this study, the anatomical success rate was $93 \%$ and $61 \%$ had functional vision improvement after MH surgery with silicone oil. The inverted ILM is usually folded into the MH. In the current study, a new technique was attempted in which the additional manipulation of the ILM remnant by folding it into the hole (inverse ILM flap) was not performed. This technique involved ILM peeling leaving a circular remnant of approximately $2 / 3^{\text {rd }} \mathrm{DD}$ of ILM attached to the margins of the macular hole. The peeled circular ILM was about 1DD width. After the ILM peeling, the existing traction on ILM got released and the entire procedure's schematic depiction is shown in Figure 7. The tangential force on the inner circular ILM (B) was towards the fovea, whereas the outer circular ILM (D) was away from the fovea. This alone relieved all the tangential tractional forces which kept the hole open preoperatively. This relieved tangential tractional force retaining the centripetal force there by facilitating the chronic macular hole to close and attain type 1 closure (93\%).

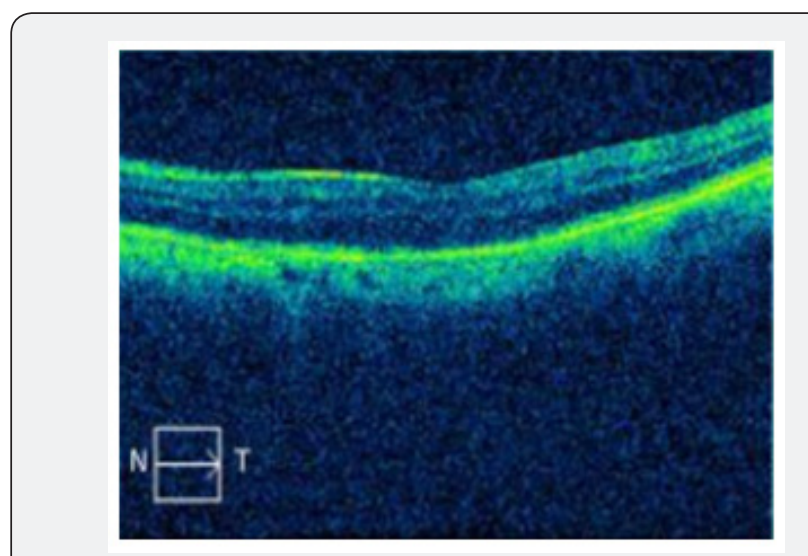

Figure 2: A sample OCT image of a patient having post-operative vision of $6 / 12$.

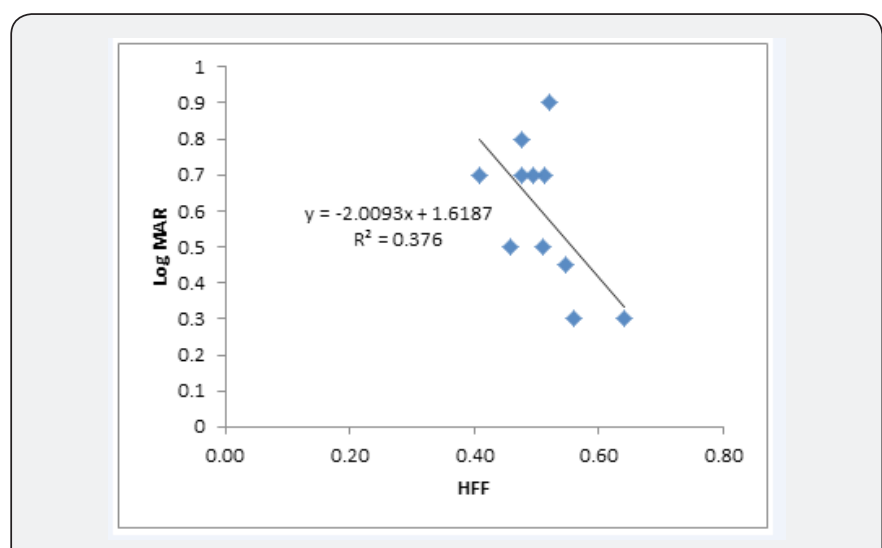

Figure 3: Correlation of HFF and 6 months post op Log MAR. 


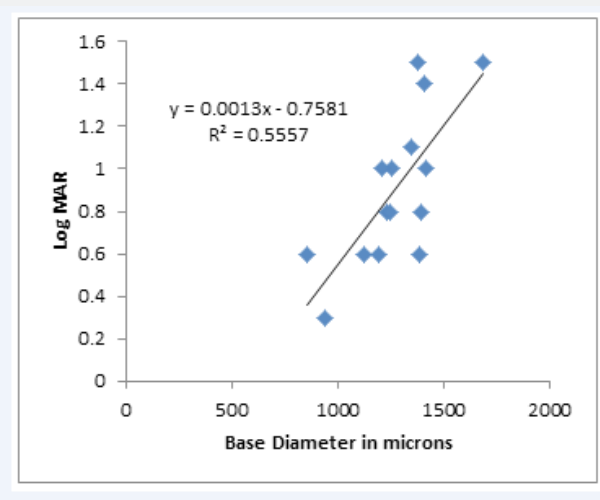

Figure 4: Correlation of Basal hole diameter and 2-week post op Log MAR.

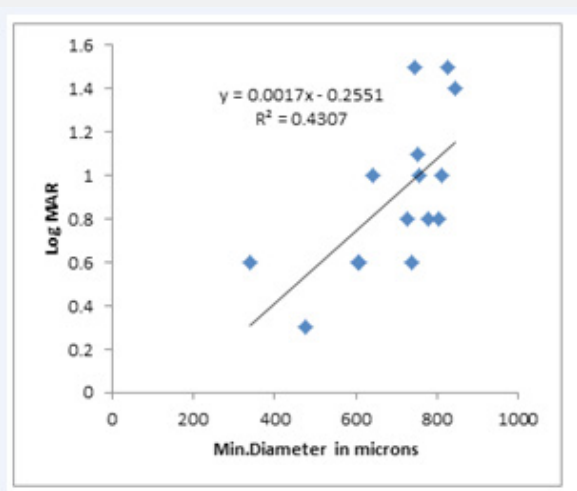

Figure 5: Correlation of Minimum diameter and 2 week post op Log MAR.

One of the subjects OCT was taken pre-operatively and immediately after 2 hours of surgery by the above-mentioned technique and is shown in Figures $8 \& 9$ respectively. Within 2 hours of the surgery, the macular hole got closed suggesting that the traction on ILM was the primary reason for the formation of the macular hole. By releasing the traction, the macular hole closed naturally. Hence this novel procedure is proposed as more appropriate surgical technique for all the chronic large diameter macular hole surgeries. In their prospective [28] randomized study of 21 consecutive patients with large idiopathic macular holes who had an unsuccessful previous surgery noted that tamponade with a mixture of silicone oil and perfluorohexyl octane (Densiron 68) was more efficacious than gas tamponade. After 12 months follow up, macular hole closure rate was $82 \%(9 / 11)$ in the $\mathrm{F}_{6} \mathrm{H}_{8}$ group and only $30 \%(3 / 10)$ of patients in the $\mathrm{C}_{2} \mathrm{~F}_{6}$ group. In this study, TRILMP technique with silicone oil tamponade achieved much higher rate of closure. In few studies, closure rates were dependent upon the minimum hole diameters, basal diameters of the $\mathrm{MH}$ and the HFF. Ullrich et al. [29] reported that a significant negative correlation was noted between both the base and the minimum diameter of the hole and the postoperative visual outcome. They concluded that higher HFF resulted in a better outcome, where an HFF of $>0.9$, the macular hole was closed following a single surgical procedure, while in eyes with $\mathrm{HFF}<0.5$ anatomical success rate was only $67 \%$. On the contrary in this work, HFF showed a significant near perfect negative correlation with Log MAR visual acuity, indicating that the postoperative visual acuity improved significantly even with a mean higher HFF value of 0.54 . Base diameter and minimum diameters also revealed moderate negative correlation with Log MAR acuity indicating that the postoperative visual acuity improved significantly despite larger diameters.

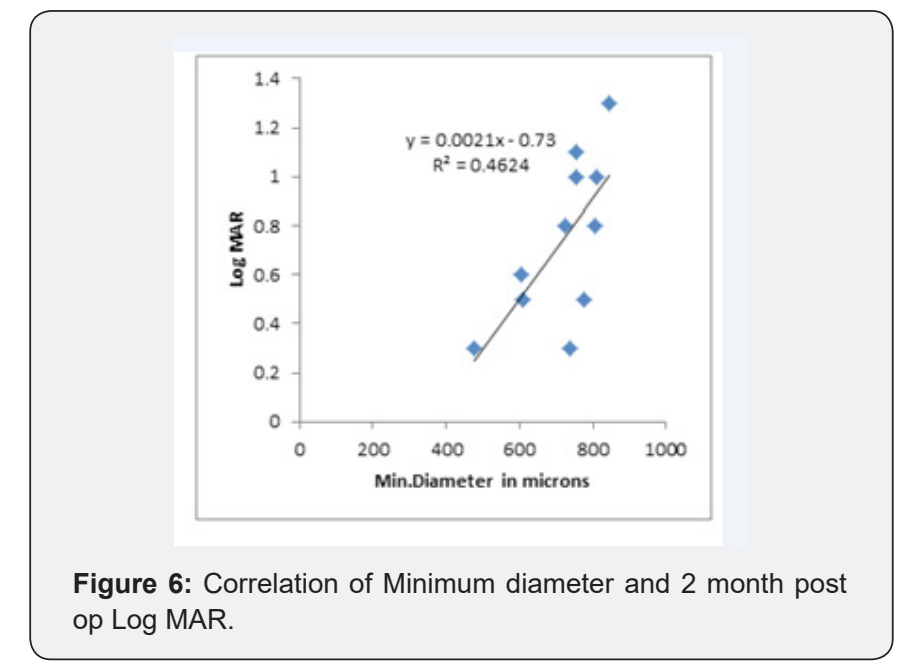

The success of silicone oil was credited to its anti-wetting properties and the ability to prevent the fluid from reaching the hole. The density of silicone oil was 3 percent less than that of the water. Hence intraocular silicone oil bubble would float to the superior position and thrust on the retina. But the floatation force exerted by silicone oil was only $1 / 30$ of that exerted by air or gas. Hence it was not the buoyancy but the hydraulic force of silicone oil which played an essential role. Silicone oil has a positive surface tension against water and forms the physical basis of the tamponade [30]. The completeness of oil fill thus plays an important role in the success rate of anatomical macular hole closure. The use of silicone oil for macular hole surgery has other advantages as well. Silicone oil tamponade is more comfortable to patients especially the elderly as no long term postoperative posturing is required. The patients can resume their normal routine lifestyle during the period of tamponade. Air travel is not contraindicated for patients with silicone oil in the eye.

The drawback in the use of silicone oil is the need for a second surgical procedure to remove the oil. The most common complication of macular hole surgery with either gas tamponade or silicone oil is cataract formation. Hence the need for a second procedure alone need not defer the surgeon from choosing silicone oil as a combined cataract silicone oil removal can be performed after 3 months. With silicon oil tamponade, a type 2 closure in the immediate post-op period got converted into type 1 closure during their subsequent follow-ups. 


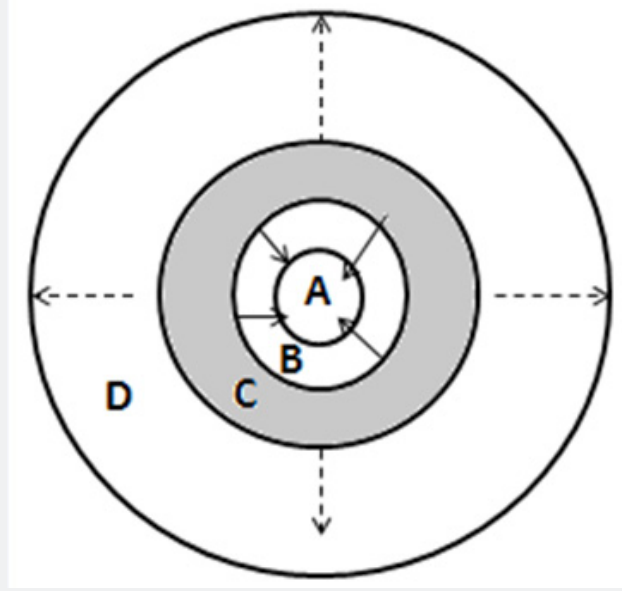

A - Macular Hole

B - Residual ILM adjacent to the hole

C - Peeled ILM

D - Peripheral intact ILM

$\uparrow$ - Tangential force of ILM towards the fovea

$\hat{\imath}$

- Tangential force of ILM away from the fovea

Figure 7: Hypothetical representation of tractional forces on ILM during TRILMP procedure.

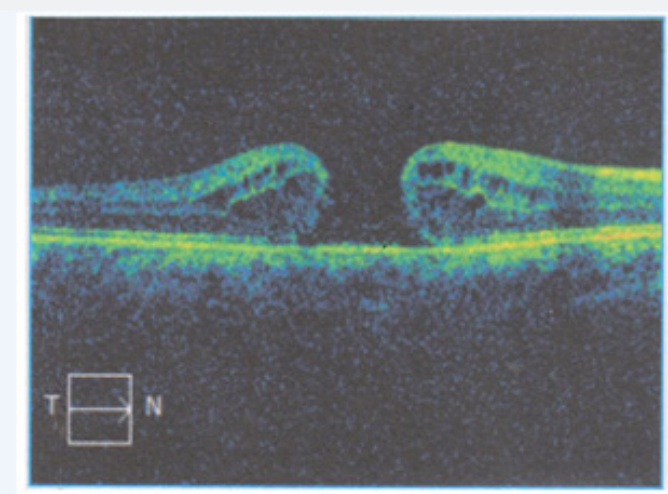

Figure 8: A sample OCT of a chronic macular hole patient before the TRILMP procedure.

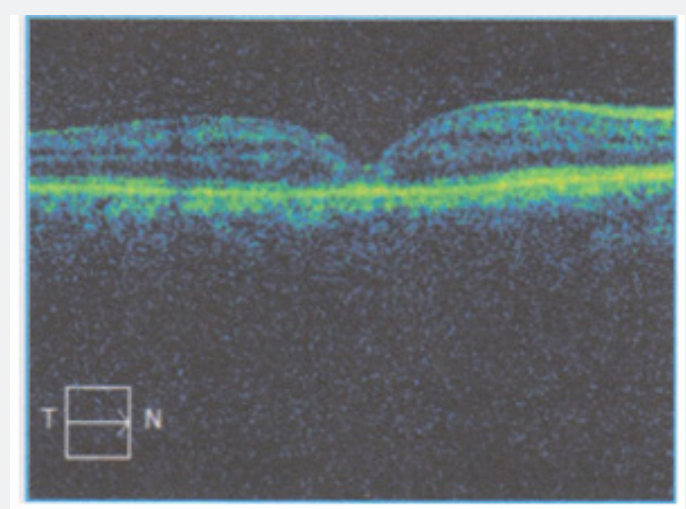

Figure 9: A sample OCT of a chronic macular hole patient after 2 hours of the TRILMP procedure.

\section{Conclusion}

A lone tamponade of silicone oil was used along with a novel surgical technique adopting TRILMP without manipulating the flap remnant around MH edge gave an enhanced anatomical and functional result even in macular holes with larger basal diameter and a medium HFF. The need of postoperative posturing was alleviated. The need of cataract surgery due to the consequence of macular hole surgery either with gas or silicone oil tamponade is 
addressed by a second surgery during which the silicone oil can be removed which has enhanced sequel compared to the other conventional approach.

\section{Acknowledgement}

Heartfelt acknowledgement to Dr. Mridula Jacob and Dr. Indu $\mathrm{G}$ for their help in literature search and manuscript preparation.

\section{References}

1. Mitchell P, Smith W, Chey T, Wang J, Chang A (1997) Prevalence and Associations of Epiretinal Membranes. Ophthalmology 104(6): 10331040 .

2. Wang S, Xu L, Jonas J (2006) Prevalence of Full-Thickness Macular Holes in Urban and Rural Adult Chinese: The Beijing Eye Study. Am J Ophthalmol 141(3): 589-591.

3. Parveen Sen, Arun Bhargava, Lingam Vijaya, Ronnie George (2008) Prevalence of idiopathic macular hole in adult rural and urban south Indian population. Clin Experiment Ophthalmol 36(3): 257-260.

4. Rahmani B, Tielsch JM, Katz J, Gottsch J, Quigley H, et al. (1996) The Cause-specific Prevalence of Visual Impairment in an Urban Population. Ophthalmology 103(11): 1721-1726.

5. (1994) Risk Factors for Idiopathic Macular Holes. Am J Ophthalmol. 118(6): 754-761.

6. Gass J (1988) Idiopathic Senile Macular Hole. Arch. Ophthalmol 106(5): 629-639.

7. Ip M (2002) Anatomical Outcomes of Surgery for Idiopathic Macular Hole as Determined by Optical Coherence Tomography. Arch Ophthalmol 120(1): 29-35.

8. Kelly N (1991) Vitreous Surgery for Idiopathic Macular Holes. Arch. Ophthalmol 109(5): 654-659.

9. Tognetto D, Grandin R, Sanguinetti G, Minutola D, Di Nicola M, et al. (2006) Internal Limiting Membrane Removal during Macular Hole Surgery. Ophthalmology 113(8): 1401-1410.

10. Glaser B, Michels R, Kuppermann B, Sjaarda R, Pena R (1992) Transforming Growth Factor- $\beta_{2}$ for the Treatment of Full-thickness Macular Holes. Ophthalmology 99(7): 1162-1173.

11. Liggett P, Skolik D, Horio B, Saito Y, Alfaro V, et al. (1995) Human Autologous Serum for the Treatment of Full-thickness Macular Holes. Ophthalmology 102(7): 1071-1076.

12. Gaudric A, Massin P, Paques M, Santiago PY, Guez JE, et al. (1995) Autologous platelet concentrate for the treatment of full-thickness macular holes. Graefes Arch Clin Exp Ophthalmol 233(9): 549-554.

13. Brooks H (2000) Macular hole surgery with and without internal limiting membrane peeling. Ophthalmology 107(10): 1939-1948.

14. Smiddy W, Feuer W, Cordahi G (2001) Internal limiting membrane peeling in macular hole surgery. Ophthalmology 108(8): 1471-1476.
15. Mester V, Kuhn F (2000) Internal limiting membrane removal in the management of full-thickness macular holes. Am J Ophthalmol 129(6): 769-777.

16. Park D, Sipperley J, Sneed S, Dugel P, Jacobsen J (1999) Macular hole surgery with internal-limiting membrane peeling and intravitreous air. Ophthalmology 106(7): 1392-1398.

17. Michalewska Z, Michalewski J, Adelman R, Nawrocki J (2010) Inverted Internal Limiting Membrane Flap Technique for Large Macular Holes. Ophthalmology 117(10): 2018-2025.

18. Kannan N, Kohli P, Parida H, Adenuga O, Ramasamy K (2018) Comparative study of inverted internal limiting membrane (ILM) flap and ILM peeling technique in large macular holes: a randomizedcontrol trial. BMC Ophthalmol 18(1): 177.

19. Narayanan R, Singh SR, Taylor S, Berrocal MH, Chhablani J, et al. (2019) Surgical outcomes after inverted internal limiting membrane flap versus conventional peeling for very large macular holes. Retina 39(8): 1465-1469.

20. Hariprasad S, Shukla S, Afshar A, Kiernan D (2014) Outcomes of chronic macular hole surgical repair. Indian J Ophthalmol 62(7): 795798.

21. Karia N, Laidlaw A, West J, Ezra E, Gregor MZ (2001) Macular hole surgery using silicone oil tamponade. Br J Ophthalmol 85(11): 13201323.

22. Kumar V, Banerjee S, Loo A, Callear A, Benson M (2002) Macular hole surgery with silicone oil. Eye 16(2): 121-125.

23. Goldbaum M, McCuen B, Hanneken A, Burgess S, Chen H (1998) Silicone oil tamponade to seal macular holes without position restrictions. Ophthalmology 105(11): 2140-2148.

24. Lai J, Stinnett S, McCuen B (2003) Comparison of silicone oil versus gas tamponade in the treatment of idiopathic full-thickness macular hole. Ophthalmology 110(6): 1170-1174.

25. Thompson J, Sjaarda R, Lansing M (1997) The results of vitreous surgery for chronic macular holes. Retina 17(6): 493-501.

26. Jaycock PD, Bunce C, Xing W, Thomas D, Poon W, et al. (2004) Outcomes of macular hole surgery: implications for surgical management and clinical governance. Eye 19(8): 879-884.

27. Ivanovska-Adjievska B, Boskurt, Jordanova-Dimovska, Semiz, Yuzer (2012) Treatment of idiopathic macular hole with silicone oil tamponade. Clin. Ophthalmol (6): 1449-1454.

28. Cillino S, Cillino G, Ferraro L, Casuccio A (2016) Treatment of persistently open macular holes with heavy silicone oil (densiron 68) versus c2f6. A prospective randomized study. Retina 36(4): 688-694.

29. Ullrich S (2002) Macular hole size as a prognostic factor in macular hole surgery. Br J Ophthalmol 86(4): 390-393.

30. Petersen J (1987) The physical and surgical aspects of silicone oil in the vitreous cavity. Graefes Arch. Clin. Exp. Ophthalmol 225(6): 452456. 
This work is licensed under Creative Commons Attribution 4.0 License

DOI: 10.19080/JOJO.2020.08.555734

\section{Your next submission with Juniper Publishers} will reach you the below assets

- Quality Editorial service

- Swift Peer Review

- Reprints availability

- E-prints Service

- Manuscript Podcast for convenient understanding

- Global attainment for your research

- Manuscript accessibility in different formats

( Pdf, E-pub, Full Text, Audio)

- Unceasing customer service

Track the below URL for one-step submission https://juniperpublishers.com/online-submission.php 